\title{
IDENTIFICACIÓN DE LOS FACTORES DE INSERCIÓN LABORAL DE LAS PERSONAS TRANS. EXPLORACIÓN DEL CASO DE LA CIUDAD DE BARCELONA
}

\author{
INDENTIFICATION OF THE FACTORS INFLUENCING THE JOB \\ INTEGRATION OF TRANSGENDER PEOPLE. EXPLORING \\ THE CASE OF BARCELONA
}

\begin{abstract}
Gerard Coll-Planas
Universitat de Vic-Universitat Central de Catalunya, España

gerard.coll@uvic.cat

Miquel Missé,

Universitat de Vic-Universitat Central de Catalunya, España miguel.misse@gmail.com
\end{abstract}

\begin{abstract}
Cómo citar / citation
Coll-Planes, Gerard y Missé, Miquel (2018) "Identificación de los factores de inserción laboral de las personas trans. Exploración del caso de la ciudad de Barcelona". OBETS. Revista de Ciencias Sociales, 13(1): 45-68.

doi: 10.14198/OBETS2018.13.1.02

\section{Resumen}

A pesar de que la población trans presenta un alto grado de exclusión laboral, no hay estudios al respecto en el contexto español. Este estudio exploratorio tiene como objetivos identificar los factores que colocan las personas trans en situaciones de vulnerabilidad frente al mercado de trabajo y establecer los subgrupos que se encuentran en una situación más vulnerable. A partir de entrevistas a personas trans e informantes clave, identificamos 9 factores (género, visibilidad, tiempos, origen y situación legal, posición socioeconómica, apoyo del entorno, nivel formativo, trabajo sexual y estado de salud) que son claves para entender las diferencias dentro del colectivo trans en relación a las posibilidades de inserción. Asimismo, establecemos tres subgrupos que se encuentran en un mayor riesgo de exclusión social.
\end{abstract}

Palabras clave: transexualidad, inserción laboral, género, exclusión social. 


\begin{abstract}
Although the trans population has a high degree of labor market exclusion, there are no studies on this topic in the Spanish context. This exploratory study aims to identify the factors that place transgender people in situations of vulnerability in relation to the labor market and establish subgroups that are in a more vulnerable situation. From interviews with key informants and transgender people, we identify nine factors (gender, visibility, time, origin and legal status, socioeconomic status, family support, educational level, sex work and health) that are key to understand the differences within the transgender population regarding work placement. Also we establish three subgroups that are at greater risk of social exclusion.
\end{abstract}

Key words: transsexuality, work placement, gender, social exclusion.

\title{
Extended abstract
}

Although the trans population presents a high level of exclusion from the labour market, no studies have been conducted in this regard in the Spanish context. This exploratory study, carried out in the city of Barcelona, seeks to identify the factors placing trans people in situations of vulnerability in the labour market and to identify those subgroups who are in a more vulnerable situation.

Based on interviews with trans people, professionals from labour insertion and key informants, we identify 9 factors that are relevant to understand the differences between trans people in terms of their insertion possibilities. The first factor identified is gender, that is to say, if the person is trans woman or trans man. In this case, trans women are in a more vulnerable position due to the gender-related salary gap, sexist discrimination in the workplace and the stigma associated with female transsexuality. The second factor is whether or not the person is visibly trans. With regard to the third factor, temporality, we identify the following dimensions: the person's age, the age at which they began their transition, the time at which they performed their transition and, finally, the current stage of their transition process. These four dimensions interact in multiple ways, generating inequalities in the employment of trans people. The fourth factor is related to the country of origin which, in the case of Barcelona, mainly affects Latin American trans women who came to the city seeking better material conditions and hoping to be able to live their gender identity with more security and respect. The origin factor can be linked to the fact that they do not have Spanish nationality, which means that the person is excluded from the possibility of changing documentation or of accessing public labour insertion programmes.

The fifth factor is the socioeconomic position, which affects essential aspects such as the educational path, the social capital when looking for work, and the financial resources to perform medical treatments (therefore, it indirectly affects health and visibility). The sixth factor is support from their surroundings (especially family), which deeply conditions how the person will experience their transition, the age at which they will publically assume their gender identity, their educational path and, indirectly, their labour insertion. The seventh 
factor, the level of training, is related to two contextual elements that influence the educational path of trans people: firstly, situations of harassment and of not feeling recognised in their gender identity; and secondly, a transphobic environment. Both can affect the choice of studies, favouring those in which the trans person believes they will be exposed to less discrimination.

The eighth factor, health, is an intermediate factor. On the one hand, it is conditioned by previous factors such as the socioeconomic position, the time at which the transition took place, the country of origin and sex work. Thus, having health problems can be a factor that hinders even more the possibility of insertion in the formal labour market. On the other hand, exclusion from the labour market can also have negative repercussions on health, culminating in eating disorders, housing problems and behavioural problems that can place integrity itself at risk. The last key factor is the performance of sex work, which is partly a product of the exclusion from the formal labour market but is also related to the fact that it is a sphere in which many trans women state that they feel valued and recognised, as well as providing them with an independent source of income. In the article, it is considered that these 9 factors are not isolated, but interact with each other, generating a diversity of positions in relation to the labour sphere.

Likewise, through fieldwork, three subgroups who are at greater risk of social exclusion are established. The first is that of trans women who perform or have performed sex work. In this group we find diverse positions: that of women who want to continue working but would like an improvement in their working conditions; that of women who want to stop sex work and join the formal labour market; and that of women who would like to combine sex work with part-time jobs or self-employment. The second subgroup is made up of visibly trans people who are job-seeking. The people interviewed, especially trans women, explain that they encounter many obstacles in the selection processes: although their $\mathrm{CV}$ is chosen, when they are called for an interview they are excluded directly if they are visibly trans or if they have not changed their documentation. The third subgroup is that of trans people whose transition takes place while they are in a job. We observe that the idea of stopping work to perform the gender transition and then re-join the labour market is widespread. The fieldwork indicates that it is a risky strategy, since looking for work as a trans person can be much more difficult than transitioning when already established in a job.

By way of conclusion, the article indicates that trans people are not, as a whole, a group at risk of social exclusion, but that there are subgroups who, depending on the combination of factors, have more or less disadvantages in the labour sphere. Consequently, the variables that interweave in each personal itinerary must be analysed. Lastly, two research lines are proposed to continue acquiring knowledge about the obstacles in trans people's access to the labour market. Firstly, it is observed that the factors identified cannot be understood independently or from an additive logic, and as such, an intersectional compression of the relation between factors would have to be broached. Secondly, the study of the problem of the labour insertion of trans people can 
be further explored both in relation to the general dynamic into which it falls (the logics of the neoliberal market) and in association with the knowledge applied which helps to move forward in the construction of knowledge that can be transferred to the public administrations, with a view to guiding their actions with respect to the professional insertion of trans people.

\section{INTRODUCCIÓN}

Las personas trans, por el hecho de no sentirse identificadas con el género atribuido al nacer en función de sus características sexuales, están expuestas a la discriminación y a la exclusión social. Esto se debe a la lógica sexista de nuestra sociedad, que se basa en la clasificación de los sujetos en mujeres y hombres atribuyéndoles diferentes roles, acceso a recursos y posiciones de poder (Izquierdo, 1998). Así, las personas trans, por no encajar en este sistema clasificatorio binario, se ven expuestas a diferentes formas de discriminación, violencia y exclusión que ponen en entredicho sus derechos de ciudadanía (Monro y Warren, 2004).

En el contexto español, la exclusión laboral de las personas trans es una temática hasta ahora prácticamente no abordada desde las políticas públicas (Platero, 2009) ni desde la investigación: apenas hay políticas dirigidas a este colectivo y no se dispone de estudios sobre su situación laboral que puedan orientar dichas políticas. Los estudios que más se acercan, se focalizan en la situación de las mujeres trans que se dedican al trabajo sexual y tampoco abordan la problemática laboral en su conjunto (Mejía, 2006; Fernández Dávila y Morales, 2011; Vartavedian, 2014). A nivel europeo sí que encontramos algunos estudios relevantes sobre la realidad de las personas trans (Whittle et al., 2007; Whittle, et al., 2008; FRA, 2014) que abordan tangencialmente el ámbito laboral y que tienen una muestra muy reducida por país, lo que impide poder sacar conclusiones diferenciadas a nivel español. Por otro lado, hay países de nuestro entorno que han llevado a cabo estudios que pueden servir como referentes aunque no abordan específicamente el tema laboral sino que hacen un planteamiento más amplio de las realidades del colectivo trans en Escocia (STA, 2008; McNeil et al, 2012), en Bélgica (De Cuypere y Olyslager, 2009; Motmans et al., 2010) o en los Países Bajos (Kuiper, 1991; Mulder, 1992; van Kesteren et al., 1996). Un estudio finés de Lehtonen y Mustola (2004), en cambio, sí que está centrado específicamente en el ámbito laboral pero analiza la situación del colectivo de lesbianas, gays, trans y bisexuales en general, siendo la muestra trans más reducida que la de los otros grupos.

Ante este vacío, y a raíz del encargo de un estudio por parte del Ayuntamiento de Barcelona, se planteó la necesidad de llevar a cabo un diagnóstico 
exploratorio que permitiera avanzar en el conocimiento sobre la situación del colectivo trans en relación a la inserción laboral para guiar el diseño e implementación de políticas municipales al respecto.

El presente artículo se propone dos objetivos. En primer lugar, determinar los factores que inciden en la trayectoria laboral de las personas trans. En segundo lugar, identificar los sectores dentro del colectivo trans que se encuentran en una situación más vulnerable en relación al ámbito laboral.

Antes de avanzar, aclaramos la terminología utilizada. Las personas cis son aquellas que viven con el género que se les ha asignado al nacer, o sea, las personas que no son trans. Consideramos como personas trans aquellas que viven en un género que no es aquel que se les ha asignado al nacer en función de sus características sexuales (independientemente de si han llevado a cabo o no modificaciones corporales o han cambiado la mención legal de sexo en su documentación). Para hacer referencia a las personas trans se usa su género de destino (con el que se identifican) y no su género de origen (el que se les asignó al nacer). Por lo tanto, los trans masculinos u hombres trans son aquellas personas que han nacido en cuerpo femenino y viven como hombres. Mientras que las trans femeninas o mujeres trans son las personas que han nacido en cuerpo masculino y viven como mujeres. Sin embargo, no todas las personas trans se identifican necesariamente con las categorías de mujer/hombre, sino que pueden tener una identificación fluida, no binaria (en algún punto del contínuum entre feminidad y masculinidad) o no sentirse identificadas con ninguna categoría genérica (Dietert y Dentice, 2009).

En el siguiente apartado presentamos un estado de la cuestión sobre la situación laboral de las personas trans. A continuación, explicamos el trabajo de campo llevado a cabo y presentamos los resultados del análisis en relación a los dos objetivos planteados. Finalmente, en las conclusiones retomamos los aspectos planteados y presentamos aspectos a tener en cuenta para la interpretación de los resultados y futuras líneas de investigación.

\subsection{Revisión de los estudios sobre la situación laboral de las personas trans}

Debido a la falta de datos sobre la situación laboral de las personas trans en el contexto español, en este apartado revisamos investigaciones llevadas a cabo sobre el tema sobre todo en el contexto europeo pero también incluimos referencias a investigaciones realizadas en el marco norteamericano que permiten dar cuenta de aspectos no estudiados en nuestro entorno.

Antes de entrar a ver cuál es la situación en los lugares de trabajo, fijémonos en los datos de personas trans que están en paro. Los estudios revisados muestran que la población trans tiene unos índices de paro claramente superiores 
a la media. Según el estudio de De Cuypere et al. (2009) llevado a cabo en Bélgica, el 37\% del grupo de mujeres trans estaban en paro, y en el caso de los hombres trans la cifra era del 35\%. En el estudio de Whittle et al. (2008), sólo el $40 \%$ de mujeres trans y el 36\% de hombres trans tenían trabajo remunerado (mientras que el Eurostat de ese mismo periodo mostraba datos de ocupación del $57,4 \%$ en el caso de las mujeres y del $72 \%$ para los hombres). En el estudio de Mulder (1992), en Holanda, el 42\% de las mujeres trans estaban en paro antes de la intervención de reasignación sexual. Seis meses después de la intervención, esta tasa había subido hasta el 62\%. Los datos para los hombres trans eran del $42 \%$ y el $45 \%$ respectivamente. A partir de los datos disponibles, se puede comprobar que las mujeres trans tienen más dificultades a la hora de encontrar trabajo. Pero, incluso para los trans masculinos la tasa de paro es inusualmente alta (Motmans et al. 2010).

Además de la transfobia en los procesos de selección de personal y en los entornos de trabajo (Whittle et al., 2007), hay otros aspectos que contribuyen a explicar este alto porcentaje de paro. Por ejemplo, Motmans et al. (2010) señalan que la discrepancia entre la identidad de género actual y la que se recoge en los certificados educativos o laborales anteriores hace que la persona trans deba decidir si mostrarlos, evidenciando su transexualidad y exponiéndose a la discriminación, o si ocultarlos y no poder poner en valor su trayectoria.

El ámbito laboral es especialmente relevante en las vidas de las personas trans por tres razones (Motmans et al. 2010). En primer lugar, porque es uno de los ámbitos que las personas trans viven como más problemáticos. En segundo lugar, porque es el que permite obtener los recursos para poder llevar una vida autónoma, algo especialmente relevante para un grupo que puede verse privado del apoyo de su entorno y que puede tener necesidad de costearse intervenciones para modificar su cuerpo. Y en tercer lugar, es especialmente relevante porque, más allá de lo material, el reconocimiento de la identidad por parte de las personas con las que se trabaja es clave en el bienestar de las personas trans.

El carácter a menudo hostil del entorno laboral hace que muchas personas trans condicionen su transición o la retrasen: el estudio de Whittle et al., (2007), por ejemplo, muestra que entre las personas trans encuestadas que no viven completamente según el género sentido, el $42 \%$ no lo hacen debido al ámbito laboral. Así, vemos que muchas personas trans no pueden desarrollarse con plenitud debido a las dificultades y la falta de reconocimiento que se encuentran precisamente en el ámbito laboral (Whittle et al., 2007). 
En cuanto a los datos sobre el porcentaje de personas trans que viven abiertamente o que esconden su identidad de género en el lugar de trabajo, la investigación de la FRA (2014) en el ámbito europeo muestra que sólo un $16 \%$ de las personas declaran ser abiertamente trans en sus puestos de trabajo; en contraposición, el $46 \%$ a menudo o siempre esconde su transexualidad.

En base a estudios llevados a cabo sobre otras identidades estigmatizadas, Law et al. (2011) plantean que ocultar aspectos importantes de la propia identidad puede implicar una presión que no te permita desarrollar tu potencial en el trabajo. Además, suele llevar a rehuir la interacción con los/las compañeros/as de trabajo a fin de evitar situaciones que puedan desvelar aquel aspecto que se quiere ocultar, lo que revierte negativamente tanto en el rendimiento como en el bienestar en el entorno laboral. Las personas que lo viven abiertamente, en cambio, se sienten liberadas de estar constantemente controlando sus acciones y pueden establecer relaciones más cómodas con compañeros/as de trabajo.

Siguiendo a Law et al. (2011), vivir abiertamente una identidad estigmatizada tiene otros efectos positivos. En primer lugar, para los compañeros de trabajo, conocer personas de un grupo estigmatizado (en este caso, personas trans) puede facilitar que sustituyan los estereotipos por una información más realista. Y en segundo lugar, para una persona que lo vive abiertamente y encuentra apoyo en su entorno laboral, mejora su capacidad de trabajo y su compromiso con su puesto de trabajo. A pesar de estos aspectos potencialmente positivos, Law et al. (2011) reconocen que en el caso de las personas trans, las investigaciones sobre las experiencias de hacer pública la identidad de género muestran que a menudo son traumáticas y comportan experiencias negativas.

El 37\% de las personas declaran haberse sentido discriminadas por ser trans en el proceso de buscar trabajo y el $27 \%$ han sido discriminadas en el entorno laboral en el último año (FRA, 2014), un porcentaje que dobla el de lesbianas, gays y bisexuales que se han sentido discriminados en el trabajo según datos del mismo estudio. Un $20 \%$ de las personas trans encuestadas en el estudio de Whittle et al. (2007) declaran haberse encontrado con esta situación. Una forma de discriminación es, por ejemplo, prohibir a las personas trans que utilicen los aseos atribuidos a su identidad de género, o hacerles utilizar los de personas discapacitadas.

En cuanto al acoso, los datos son variables porque se aplican a diferentes contextos, tienen diferentes muestras y, sobre todo, porque las preguntas se han formulado de formas no comparables. En el estudio de Motmans et al. (2010), llevado a cabo en Bélgica, un 14\% de las personas trans declaran haber 
sido acosadas en su puesto de trabajo actual. Se trata de una cifra similar al 15\% de personas de la encuesta europea de la FRA (2014) que responden que han vivido conductas o comentarios negativos en el trabajo en los últimos 5 años.

Estos datos son superiores si no sólo se pregunta por el puesto de trabajo actual o por los últimos 5 años sino por el conjunto de la trayectoria laboral. Este es el caso de un estudio llevado a cabo en Escocia, según el cual el 53\% personas encuestadas han recibido alguna forma de acoso (STA, 2008); o del de Whittle et al. (2007), a nivel europeo, que muestra que el 39\% de las personas trans han experimentado acoso verbal (comentarios, insultos) mientras que un porcentaje inferior han recibido amenazas y agresiones físicas.

Donde sí hay consenso en los estudios revisados es en que la transición género es el momento más delicado, en el que hay más riesgo de discriminación y acoso (Motmans et al. 2010). De hecho, Whittle et al. (2007) plantean que muchas personas trans tienen trayectorias laborales positivas hasta el momento de empezar a vivir según la identidad de género sentida. Entre los factores que dificultan la transición están los estereotipos tanto de empleadores/as como de compañeros/as de trabajo y su falta de conocimiento sobre cómo acompañar la persona que está haciendo la transición, lo que puede llevar a reacciones de hostilidad y aislamiento (Budge, 2010). El estudio de Barclay y Scott (2006) se aproxima a las reacciones del entorno laboral a partir del estudio de caso de una mujer transexual en el Reino Unido, mostrando desde una forma más micro las reacciones que suscita este hecho en las diferentes personas de su trabajo como no dejar usar el baño del género sentido o no referirse a ella con el nombre o la identidad de género sentida.

Debido a este clima laboral a menudo hostil, el 23\% de las personas encuestadas en el estudio de Whittle et al. (2007) declaran que se han sentido obligadas a dejar su puesto de trabajo a raíz de su transición o lo han dejado porque no sentían que sus empleadores/as les dieran apoyo en la transición. Según el estudio de Scottish Transgender Alliance (STA, 2008), el 8\% de las personas encuestadas declaran haber sido despedidas por ser trans y un 13\% dicen que han tenido que dejar su puesto de trabajo al menos una vez debido al miedo de una posible discriminación o acoso.

El contexto transfóbico descrito hasta ahora nos podría explicar porque, como apunta un estudio finlandés (Lehtonen y Mustola, 2004), las personas trans tendrían más tendencia al autoempleo. Así, las personas trans están más inclinadas a escoger formas de trabajo autónomas, ya que ofrecerían más libertad a la hora de desarrollar su identidad de género.

En los estudios revisados se apuntan tres factores que generan diferencias en las posibilidades de discriminación. El primero es el hecho de ser trans 
masculino, femenina o no binario. Las investigaciones revisadas coinciden en que las mujeres trans suelen estar expuestas a más discriminación y acoso (Whittle et al., 2007; FRA, 2014). A raíz de la transición, tienen más probabilidades de experimentar acoso, pérdida de confianza por parte de los/las empleadores/as, descenso en la jerarquía laboral, reducción de la retribución, y despido (Schilt y Wisawll, 2008; Law et al., 2011). En el caso de los trans masculinos, la transición puede incluso conllevar la atribución de una mayor autoridad, mejor trato personal, más respeto, mejores evaluaciones, más retribución, y posibilidades de promoción (Schilt, 2006; Schilt y Wisawll, 2008). Por otra parte, en el estudio de Schilt y Connell (2007) se muestra que los informantes que no se identifican de forma binaria tratan de conformar una identidad femenina o masculina para reducir la conflictividad en el trabajo y poder mantener el empleo.

El segundo factor de discriminación es el hecho de ser visiblemente trans. Las personas que pasan desapercibidas físicamente, que tienen la documentación cambiada y que deciden no comunicar su transexualidad tienen menos problemas de inserción laboral que las personas que son visibles (Dieter y Denticer, 2010). Este factor se suele solapar con el anterior, ya que debido a las características corporales y efectos de los tratamientos médicos, a las mujeres trans les suele costar más pasar desapercibidas e invisibilizar su trayectoria. Esto se debe en parte a la misma estructura y forma corporal (voz, altura, tamaño de las manos, la nuez del cuello...) y a que la terapia hormonal suele tener menos efecto en las mujeres trans que en los hombres trans (WPATH, 2012). Es por ello que de los trans masculinos encuestados en el estudio de Schilt y Wisawll (2008), llevado a cabo en Estados Unidos, un 56\% responde que siempre es percibido como hombre, mientras que solo un $17 \%$ de las mujeres trans son siempre reconocidas en función de su género sentido.

El tercer y último factor que destacan los estudios es el tipo de trabajo. El estudio de la FRA (2014) apunta que las situaciones de discriminación y acoso son reportadas más frecuentemente por las personas con menos ingresos, por tanto, que tienen trabajos menos cualificados. En la misma línea, Schilt y Wisawll (2008) muestran que los casos de situaciones más violentas los sufren mujeres trans en trabajos no cualificados.

En relación al papel de las empresas, las personas trans que participaron en el estudio de STA (2008), en Escocia, respondieron que el departamento de Personal / Recursos Humanos era el servicio del que estaban menos satisfechos. El $40 \%$ de las personas trans encuestadas evaluaban el trato recibido en este departamento como "extremadamente malo". Según el mismo estudio, los departamentos de Personal / Recursos Humanos no cumplen sus obli- 
gaciones respecto a las leyes antidiscriminatorias y, de hecho, perpetúan la discriminación en vez de prevenirla.

Según Whittle et al. (2007), los/las empleadores/as no desarrollan los procesos adecuados para apoyar a trabajadores/as trans. De hecho, el 15\% de personas encuestadas en el estudio de STA (2008) declaran que el/la empleador/a falló en proteger su privacidad en relación a su transexualidad y esto se tradujo en discriminación y acoso laboral. Incluso algunas personas trans expresan que han acabado abandonando su trabajo debido a la incompetencia de sus empleadores/as a la hora de acompañarlos en su transición de género (Whittle et al., 2007).

\section{METODOLOGÍA}

El carácter exploratorio de esta investigación requiere una aproximación metodológica cualitativa, ya que no se pretende buscar regularidades estadísticas sino identificar vivencias y percepciones (Ruiz Olabuénaga, 1996). Sin embargo, la identificación de los factores que proponemos en el presente artículo puede guiar futuras investigaciones que adopten métodos cuantitativos para identificar estadísticamente el peso y la relación entre factores.

El trabajo de campo del artículo se ha basado en 28 entrevistas semidirigidas. La muestra de la investigación se pueden clasificar en tres categorías. En primer lugar, entrevistas a 12 personas trans que viven en Barcelona. A pesar de no ser una muestra representativa, se ha buscado que presentaran diferentes perfiles en relación a la edad, el origen, la situación laboral y el ejercicio del trabajo sexual. Las personas entrevistadas fueron contactadas a través de las redes personal del equipo de investigación y de agentes que se dedican a la inserción laboral de personas trans.

Respecto a esta muestra hay que tener en cuenta que: se ha priorizado a las mujeres trans porque son las que concentran más problemáticas; ninguna de las personas entrevistadas presenta una identidad no binaria; y las tres personas entrevistadas migrantes provienen de países de América Latina (México, Ecuador y Venezuela).

En segundo lugar, se ha entrevistado a 5 informantes clave que tienen un conocimiento de la situación de las personas trans debido a su actividad profesional:

- Profesional de la medicina especializada en transexualidad (referida como 'médica')

- Psicóloga especializada en diversidad de género y en trabajo sexual que trabaja en el ámbito privado ('psicóloga 1') 
Tabla 1. Relación de personas entrevistada trans

\begin{tabular}{l|l|l|l|l}
\hline Pseudónimo & $\begin{array}{l}\text { Identidad } \\
\text { de género }\end{array}$ & Edad & Origen & $\begin{array}{l}\text { Situación laboral en el } \\
\text { momento de la entrevista }\end{array}$ \\
\hline David & Masculino & $18-24$ & Autóctono & Técnico en emergencias sanitarias \\
\hline Iris & Femenina & $25-34$ & Autóctona & Coordinadora de tienda de comida rápida \\
\hline Mike & Masculino & $25-34$ & Autóctono & Trabajo temporal en el ámbito del turismo \\
\hline Amanda & Femenina & $25-34$ & Migrante & Trabajadora sexual y profesora de inglés \\
\hline Pau & Masculino & $35-44$ & Autóctono & En el paro, con prestación \\
\hline Maite & Femenina & $35-44$ & Autóctona & $\begin{array}{l}\text { Trabajo a tiempo completo como } \\
\text { informática }\end{array}$ \\
\hline Mónika & Femenina & $35-44$ & Autóctona & $\begin{array}{l}\text { En el paro, cobrando Renta Mínima } \\
\text { de Inserción }\end{array}$ \\
\hline Alina & Femenina & $35-44$ & Migrante & Trabajadora sexual \\
\hline Sara & Femenina & $35-44$ & Migrante & $\begin{array}{l}\text { Trabajo a tiempo parcial y temporal } \\
\text { fruto de programa de inserción }\end{array}$ \\
\hline Eva & Femenina & $35-44$ & Autóctona & Trabajadora sexual \\
\hline Raquel & Femenina & $45-54$ & Autóctona & En el paro, sin prestación \\
\hline Maria & Femenina & $45-54$ & Autóctona & $\begin{array}{l}\text { Combina dos trabajos a tiempo parcial y } \\
\text { temporales fruto de programa } \\
\text { de inserción }\end{array}$ \\
\hline
\end{tabular}

- Psicóloga especializada en diversidad de género que ejerce desde el ámbito público ('psicóloga 2')

- Dos técnicas de una asociación que trabajan dando apoyo a trabajadoras sexuales ('técnica 1', 'técnica 2')

En tercer lugar, se realizaron 11 entrevistas a agentes que están implicados en la inserción laboral de personas trans (en el diseño o la implementación de programas, en la gestión de servicios o en fomentando la contratación). Estas entrevistas se pueden clasificar en dos subcategorías:

- Personal del Ayuntamiento de Barcelona: Directora de Equidad Social y Salud (dir. Equidad); Coordinadora de Programas de inserción socio-laboral de Barcelona Activa (coord. Inserción); responsable de Programas de formación y experiencia laboral de Barcelona Activa (coord. Formación); Coordinadora de la Red de Economía Social de Barcelona Activa (coord. Red); Técnica del Programa Trans-Ocupación en Barcelona Activa (téc- 
nica Trans-Ocupación); Responsable del Plan LGTB (resp. Plan); y la Responsable de ABITS, Agencia para el Abordaje Integral del Trabajo Sexual (resp. ABITS).

- Entidades sociales que participan en la inserción laboral trans: representante de 3 entidades gestionan servicios de inserción laboral que específicos o que incluyen a personas trans y que tienen convenios con el Ayuntamiento de Barcelona para llevarlos a cabo (rep. Entidad 1, Entidad 2, Entidad 3) y representante entidad LGTB participa en la Red de Economía Social de Barcelona Activa (rep. Entidad LGBT).

Las entrevistas tenían una duración de unos 45 minutos y tenían como objetivo identificar las principales problemáticas de las personas trans en relación al ámbito laboral, evaluar el modelo actual de inserción laboral de la ciudad de Barcelona y recoger propuestas de mejora.

Las transcripciones literales de las entrevistas fueron volcadas en una matriz Excel para permitir su categorización y se analizaron mediante la metodología de análisis de contenido, basada en una lectura sistemática, replicable, y válida de los datos, con el objetivo de interpretar los datos de acuerdo a su contexto y dar cuenta de su sentido manifiesto y latente (Andréu, 2002).

\section{RESULTADOS Y DISCUSIÓN}

\subsection{Identificación de los factores}

En este apartado sintetizamos las principales conclusiones de la investigación en relación a la identificación de factores principales que inciden en la inserción laboral del colectivo trans. Por razones de espacio, en este artículo no se podrá profundizar en el análisis de las entrevistas, sino que se focalizará la atención en la síntesis de los resultados obtenidos.

La primera conclusión del estudio llevado a cabo es que el hecho de ser trans es un factor que puede dificultar el acceso al mercado de trabajo pero no es en sí mismo un factor de exclusión laboral: la trayectoria laboral de una persona trans es el resultado de la combinación de muchos factores que se interseccionan de una forma compleja (Platero, 2013; Coll-Planas y Cruells, 2013). A continuación se examinan los principales.

\section{Género}

El primer factor relevante es si la persona es trans femenina o trans masculino. A la brecha salarial de género y las discriminaciones sexistas que se encuentran las mujeres en el mercado de trabajo (Torns y Recio, 2012) el hecho de ser trans 
aún las sitúa en peores condiciones, habiendo un amplio consenso entre las personas entrevistadas y entre los estudios previos revisados (Whittle et al., 2007; Schilt y Wisawll, 2008; FRA, 2014). Parte de las dificultades proviene de los estereotipos alrededor de la transexualidad femenina (asociación con el trabajo sexual, con la falta de formalidad, con la marginación...) (Ent. Psicóloga 1, rep. Entidad 3), mientras que la transexualidad masculina es más invisibilizada y no presenta el mismo nivel de estigma (Missé, 2013). Como veremos, el género afecta además a otros factores que abordamos a continuación.

\section{Visibilidad}

El hecho de ser visiblemente trans es otro factor clave que condiciona la posibilidad de pasar procesos de selección de personal (Ent. Eva, resp. Plan, Psicóloga 1 y Psicóloga 2). La visibilidad depende de factores legales (el hecho de haber efectuado o no el cambio de la mención de sexo legal y del nombre), físicos y hormonales (como son la apariencia o la voz). En general a las mujeres trans les suele ser más difícil pasar desapercibidas, mientras que los chicos trans suelen ser más indistinguibles (Ent. Médica, Psicóloga 1 y Psicóloga 2). De hecho, una vez hecha la transición, los tres trans masculinos entrevistados afirman pasar desapercibidos como tales y escogen con quien quieren compartir esta información, algo que no sucede en el caso de las trans femeninas.

Más allá de la identidad de género, la visibilidad también puede estar relacionada con la edad en que se ha hecho la transición, los recursos que se han destinado a la modificación corporal o la propia constitución corporal. La visibilidad de ser trans, sin embargo, no se debe sólo a la posibilidad del passing (Halberstam, 2005), esto es, de pasar desapercibido/a: sino que la visibilidad de la trayectoria trans también puede deberse a una voluntad política de evidenciar la propia transición para cuestiona el cisgenerismo, que presupone que todas las personas se identifican con el género atribuido al nacer en función del sexo (Missé, 2013).

\section{Tiempos}

En este factor hemos agrupado cuatro aspectos que tienen que ver con la temporalidad. El primero es la edad actual, que afecta en las posibilidades de encontrar trabajo. En este sentido, las mujeres trans mayores presentan una especial vulnerabilidad, especialmente a partir de los 45 años, acentuando así la dinámica general edadista del mercado laboral (Serrano, 2011). Hay que tener en cuenta que en el contexto español hay pocos hombres trans mayores, ya que 
es a partir de la década de 1990 cuando se inician los primeros tratamientos médicos de modificación corporal masculinizadora, se extiende la categoría 'trans masculino' y empiezan a aparecer personas que se identifican en ella (Coll-Planas, 2010; Soley-Beltran y Coll-Planas, 2011).

El segundo aspecto es el momento de la transición, es decir, en qué estadio se encuentra la persona en relación a su proceso de vivir según la identidad de género sentida (si ha iniciado tratamientos hormonales, intervenciones quirúrgicas, a qué personas de su entorno lo ha comunicado, etc.). Hay que tener en cuenta que, como hemos visto, el inicio de las transiciones es el momento más frágil a nivel personal y de relación con el entorno (incluido el laboral y el formativo) (Motmans et al. 2010; Whittle et al., 2007). En este sentido, las personas trans entrevistadas identifican haberse sentido más vulnerables y más discriminadas en el período en que estaban transitando y su físico no les permitía ser identificadas con el género con el que se identifican.

El tercer aspecto es la edad en que la persona hizo o hace la transición: cuanto más joven se inicie la transición más probable es que los tratamientos (hormonales o quirúrgicos) generen un mayor cambio en la apariencia y una mayor posibilidad de invisibilidad (Ent. Médica).

El último aspecto es la generación. Es un factor que puede ir relacionado con el anterior pero no necesariamente: una mujer trans de 40 años, por ejemplo, puede que hiciera la transición a los 20 años o que la esté iniciando en este momento de su vida (es el caso de Maria y Mónika respectivamente). Este aspecto genera diferencias. Por un lado, haber hecho la transición de joven puede implicar que la persona haya tenido más tiempo para modificar su cuerpo y para pasar desapercibida pero, al mismo tiempo, supone haber llevado a cabo la transición en momentos menos favorables a nivel de: aceptación social y familiar; posibilidades de inclusión formativa y laboral; acceso a los servicios de salud; y de situación legal (las personas trans que hicieron su proceso en los años 1960 y 1970 han vivido a menudo experiencias de persecución política). Por otro lado, hacer la transición de mayor puede implicar haber vivido gran parte de la vida sin sentirse a gusto con uno/a mismo/a y que sea más difícil transformar los rasgos físicos o la voz; pero al mismo tiempo puede implicar que la persona haya podido desarrollar una trayectoria educativa y laboral normativa que la coloque en una mejor posición respecto a la inserción laboral. Esto coincide con las conclusiones del estudio de Lombardi et al. (2001), según el cual las personas trans mayores, empleadas a tiempo completo y con niveles salariales más altos era menos probable que experimentaran un impacto económico adverso a raíz de su transición. 
Origen y situación legal

Barcelona se ha convertido en una ciudad de llegada de mujeres trans principalmente latinoamericanas que buscan mejores condiciones de vida y poder vivir su identidad de género con más seguridad y respeto (Mejía, 2006; Fernández Dávila y Morales, 2011; Vartabedian, 2014). En ocasiones, en sus países de origen pueden haber sido víctimas de violencia y persecución por ser trans y haberse sometido a procesos de modificación corporal no controlados que pueden haber derivado en problemas de salud (Ent. Psicóloga 1, Médica y rep. Entidad LGTB).

En relación a la dimensión jurídica, hay que tener en cuenta que no tener la nacionalidad española excluye de la posibilidad de hacer el cambio de nombre (Platero, 2009), lo que expone a la discriminación porque evidencia la no correspondencia entre la identidad de género vivida y la legal. Además, las personas sin permiso de residencia y/o trabajo quedan excluidas de los servicios de inserción laboral (Ent. rep. Entidad 2, Entidad LGTB, coord. Inserción).

\section{Posición socioeconómica}

Se trata de una variable que afecta a aspectos fundamentales como la trayectoria formativa, el capital social al buscar trabajo, o los recursos económicos para hacer tratamientos médicos (por lo tanto, incide indirectamente en la salud y en la visibilidad). A pesar de su centralidad, por si misma no resulta explicativa ya que, como muestra nuestro trabajo de campo, algunas entrevistadas que proceden de clases acomodadas y que han podido obtener formación postobligatoria se encuentran en paro o dependiendo de trabajos temporales que han obtenido gracias a la participación en programas de inserción (este es el caso de Maria y Mónika).

\section{Apoyo del entorno}

El apoyo del entorno (especialmente familiar) desde un punto de vista afectivo aparece en las entrevistas como aún más fundamental que el económico para favorecer la trayectoria formativa y laboral (Ent. Psicóloga 1 y Psicóloga 2). De hecho, el apoyo del entorno es un factor que condiciona profundamente cómo la persona vivirá su transición, la edad en que asumirá públicamente su identidad de género, su trayectoria formativa e, indirectamente, su inserción laboral.

Nivel formativo

La trayectoria educativa de las personas trans puede verse afectada de dos formas. En primer lugar, situaciones de acoso, no sentirse reconocidas en su iden- 
tidad de género o estar centradas en llevar a cabo la transición puede conducir a un bajo rendimiento académico o incluso al abandono de los estudios (Ent. Psicóloga 1, Psicóloga 2). En segundo lugar, un entorno transfóbico puede influenciar la elección de estudios favoreciendo aquellos en que la persona trans considere que recibirá menos discriminaciones (en el caso de las trans femeninas, por ejemplo, el ámbito de la estética). De hecho, este mismo sesgo se observa en relación a los cursos de inserción laboral para mujeres trans, que van orientados a profesiones feminizadas como la estética o las tareas del hogar (Ent. rep. Entidad 1, Entidad 3 y coord. Formación). Esta orientación coincide con las expectativas de algunas de las mujeres trans, como Raquel y Sara, que antes de la transición se dedicaban al mundo de la construcción pero no quieren volver a él porque sienten que cuestionaría su feminidad. Sin embargo, Maite y Amanda tienen intereses laborales que escapan de los estereotipos femeninos (informática y mecánica respectivamente) y critican el tipo de oportunidades que ofrecen los programas de inserción laboral.

En cualquier caso, hay que decir que la exclusión laboral que vive parte del colectivo no se explicaría principalmente por la falta de formación (Ent. rep. Entidad LGBT): a lo largo del trabajo de campo hemos podido encontrar mujeres trans con una amplia trayectoria formativa pero sin posibilidad de encontrar trabajo al margen del trabajo sexual o los programas de inserción. Este es el caso de Sara, con formación de ingeniera de telecomunicaciones, o de Maria con un Máster en Dirección y Administración de empresas.

\section{Trabajo sexual}

El ejercicio del trabajo sexual es un producto en parte de la exclusión del mercado laboral formal pero también está relacionado con el hecho de que es un ámbito en el que muchas mujeres trans afirman sentirse valoradas y reconocidas además de proveerles de una fuente de recursos económicos de forma autónoma, ya que no suelen trabajar por cuenta ajena (Juliano, 2004; Mejía, 2006; Vartabedian, 2014).

El trabajo sexual las expone a la presión policial, las puede hacer entrar en dinámicas de drogodependencia, debido a la falta de regulación no les permite cotizar y cuando quieren dejar de ejercer el trabajo sexual (por razones de salud, de edad o porque quieren buscar otras opciones) les puede costar encontrar alternativas en el mercado laboral formal (Ent. Eva, Técnica 1, Técnica 2, Psicóloga 1, rep. Entidad 3).

Se observa un cambio en el perfil de mujeres trans que se dedican al trabajo sexual en Barcelona, producto de dos fenómenos (Ent. Eva, Entidad 3, Psicóloga 1, resp. ABITS). En primer lugar, el mayor apoyo que encuentran 
las chicas trans autóctonas por parte de su entorno familiar y las mayores posibilidades de inserción laboral hacen que muchas de ellas no vean el trabajo sexual como una opción a valorar para obtener ingresos. En segundo lugar, a partir del año 2000 se observa un importante flujo de mujeres trans inmigrantes procedentes mayoritariamente de América Latina que vienen a Barcelona en busca de espacios más respetuosos con su identidad y que encuentran como principal opción dedicarse al trabajo sexual (Mejía, 2006; Vartabedian, 2014). La confluencia de estos dos aspectos haría que la población de mujeres trans que actualmente se dedican al trabajo sexual esté compuesta por mujeres trans autóctonas de más de 35 años pero, sobre todo, por mujeres trans latinoamericanas con edades más diversas.

Estado de salud

Se trata de un factor intermedio. Por un lado, está condicionado por factores anteriores como la posición socioeconómica (que puede garantizar el acceso a mejores tratamientos de modificación corporal), el momento en que se realiza la transición (con mayor o menor control de los procesos de transición), el país de origen (donde los tratamientos pueden ser más o menos controlados implicando diferentes niveles de riesgo para la salud) y el trabajo sexual (mayor riesgo de VIH, de drogodependencia, etc.) (Ent. Médica, Psicóloga 1 y rep. Entidad LGBT). Así, tener problemas de salud puede ser un factor que dificulte aún más la posibilidad de inserción en el mercado laboral formal.

Por otra parte, la exclusión laboral también puede repercutir negativamente en la salud, desembocando en problemas de alimentación, de vivienda y de conductas que pueden poner en riesgo la propia integridad (Espluga et al., 2004).

\section{Relación entre los factores}

A la hora de interpretar el listado de factores apuntado en el apartado anterior se debe tener en cuenta que no todos tendrían el mismo peso ni se pueden entender de forma independiente, como una lista de factores a sumar siguiendo una lógica aditiva) (Platero, 2013).

En las relaciones entre los factores se puede distinguir entre: primarios (que figuran arriba del todo en el Gráfico 1), que pueden tener relaciones causales entre ellos; factores intermedios (que están en la parte media del gráfico), que serían producto de la combinación de los primarios; y, finalmente, la inserción laboral que es resultado de la combinación de los factores primarios y secundarios y que puede influir, a su vez, en aspectos como la salud o el ejercicio del trabajo sexual. 


\section{Gráfico 1. Relación entre los factores}

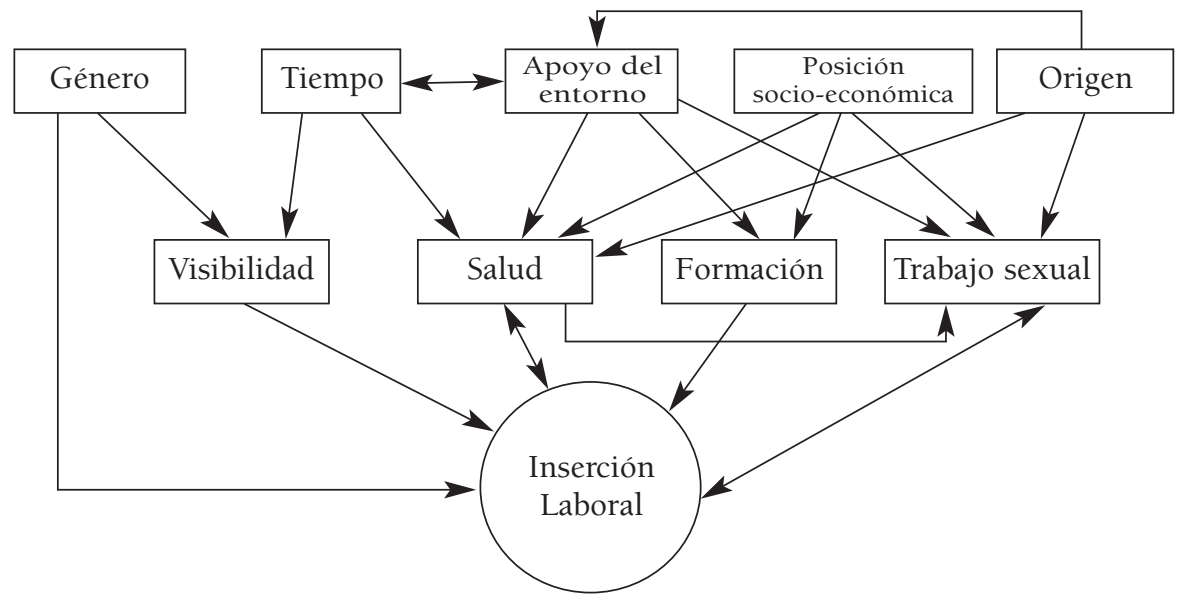

\subsection{Identificación de grupos vulnerables}

A continuación presentamos brevemente los principales rasgos de los perfiles que se encuentran en una situación de mayor vulnerabilidad en relación a la inserción laboral.

Mujeres trans que ejercen o han ejercido trabajo sexual

Dentro de este grupo, hay que tener en cuenta que las posiciones respecto al ejercicio del trabajo sexual y la inserción en el mercado laboral formal pueden variar (Ent. Psicóloga 1, Técnica 1, Técnica 2, resp. ABITS, rep. Entidad 2 y Entidad 3). Las diferentes posiciones las podemos clasificar en tres grupos:

- Las mujeres trans que quieren seguir ejerciendo el trabajo sexual, que reclaman menos presión policial para poder ejercerla en mejores condiciones y que no están interesadas en las medidas de inserción laboral.

- Las mujeres que por el momento no quieren dejar el trabajo sexual pero quieren explorar otras posibilidades para compaginarlo o para tener alternativas en el momento en que lo quieran dejar. Este grupo está interesado en medidas de inserción laboral pero siempre y cuando no requieran el abandono del trabajo sexual (como establecen la mayoría de programas evaluados en su momento en Barcelona) y no impliquen un compromiso muy fuerte en cuanto a dedicación.

- Las que quieren dejar (o se ven obligadas a dejar) el trabajo sexual porque se han visto abocadas al trabajo sexual sin haber querido dedicarse 
a ello ${ }^{1}$, porque ya no les sale a cuenta (por la caída de ingresos, porque tienen menos clientes debido a la edad) o porque no se pueden dedicar por motivos de salud. Este grupo es el que está más interesado en las medidas de inserción laboral. Dentro de este grupo, un subgrupo que es especialmente vulnerable y que requiere una atención más allá de la formación laboral es el de las mujeres trans que dejan el trabajo sexual debido a la edad, que no tienen formación ni experiencia laboral previa en el mercado formal y que no han cotizado, por lo que no tienen derecho a prestación de desempleo ni de jubilación.

En estos dos últimos subgrupos hay una serie de factores que dificultan que encuentren trabajo en el mercado formal: la visibilidad como trans, el estigma asociado al trabajo sexual, la falta de formación previa, la inexistencia de experiencia laboral anterior en el mercado formal, las expectativas no realistas en relación a las ganancias (viniendo de un sector en el que suelen ganar más dinero de lo que pueden obtener en las nuevas tareas que se les ofrecen) y la inserción en determinadas dinámicas del trabajo sexual (lógica de la inmediatez, posibilidad de drogodependencia, etc.).

Personas trans visibles que buscan trabajo

Las personas trans visibles (por la apariencia, la voz o porque no han modificado su documentación) están expuestas a discriminación al entrar en procesos de búsqueda de trabajo. En este sentido, las personas entrevistadas, especialmente las mujeres trans, explican que se encuentran muchos obstáculos en los procesos de selección: aunque hayan escogido su currículum, al citarlas a la entrevista se las excluye directamente si son visiblemente trans o si no tienen la documentación cambiada (Ent. Amanda, Eva, Mónika y Sara).

Personas trans que hacen su transición en el puesto de trabajo

Nuestro trabajo de campo coincide con los resultados de otros estudios llevados a cabo (Motmans et al. 2010; Whittle et al., 2007), en el sentido de apuntar que la transición en el trabajo es un momento de especial vulnerabilidad. En el caso de tener trabajo antes de hacer la transición, las personas entrevistadas han desarrollado tres estrategias. La primera es dejar el trabajo con el objetivo de centrarse en la transición y luego buscar otro, ya con la nueva identi-

\footnotetext{
${ }^{1}$ En el trabajo de campo encontramos los dos extremos: Maria recurre al trabajo sexual fruto de la desesperación mientras que Amanda es activista por los derechos de las trabajadoras sexuales y afirma ejercerlo voluntariamente.
} 
dad. Esta opción supone un alto riesgo por la dificultad de encontrar trabajo siendo trans (especialmente para las mujeres y si son visiblemente trans). Esta es la estrategia adoptada por Maria, que deja un cargo directivo en una gran empresa para hacer la transición y abrir después una tienda propia (que quebrará debido a los prejuicios de clientes y trabajadores y por la coincidencia con la crisis, abocándola al trabajo sexual).

La segunda estrategia es cambiar de trabajo por otro donde consideran que podrán hacer la transición de forma más segura. Es el ejemplo de Steve que, teniendo estudios universitarios, deja su trabajo cualificado para ir a fregar platos en un hotel ya que prioriza estar en un entorno facilitador que no el tipo de trabajo. Una vez hecha la transición en este contexto, vuelve a buscar trabajos acorde con su formación previa.

Finalmente, encontramos personas que hacen la transición en el puesto de trabajo. Este es el caso de: Iris, que empieza a vivir como mujer desde su puesto de jefa de una tienda de comida rápida; y de Maite, que opta por ir haciendo un cambio paulatino (dejarse crecer el pelo, vestir más andrógina) mientras decide bajar de categoría para tener un puesto de trabajo más de oficina, en que esté menos en contacto con clientes.

En este sentido, los estudios revisados apuntan que el mercado laboral es menos hostil con las personas trans ya conocidas que a la hora de contratar personas trans desconocidas. Es decir, que la estrategia que intuitivamente siguen diversas personas trans de dejar de trabajar para transitar es en muchos casos más arriesgada que transitar en el puesto de trabajo que la persona ya tiene.

\section{CONCLUSIONES}

Uno de los objetivos de este artículo era identificar los factores clave que inciden en la inserción laboral del colectivo trans. Esta aproximación, de carácter exploratorio, nos ha permitido acercarnos a la delimitación de las variables que son relevantes para entender de una forma más precisa la diversidad dentro del colectivo en relación a sus posibilidades de inserción laboral. Esto nos ayuda a entender que las personas trans no son, en conjunto, un colectivo en riesgo de exclusión sino que hay subgrupos que, en función de la combinación de factores, tienen más o menos desventajas en el ámbito del trabajo.

A partir de este estudio exploratorio apuntamos dos líneas de investigación que permitirían avanzar en el conocimiento de la problemática. En primer lugar, consideramos que los factores identificados no se pueden entender independientemente ni desde una lógica aditiva. En este sentido, habría que 
avanzar hacia una compresión interseccional de la relación entre factores. Esto implica entender que la convergencia entre los diferentes factores genera nuevas realidades y desigualdades que no se pueden deducir de una simple suma de situaciones aisladas (Platero, 2013; Coll-Planas y Cruells, 2013).

En segundo lugar, la problemática de la inserción laboral de las personas trans puede profundizarse tanto en relación a la dinámica general en la que se inserta como en vinculación con el conocimiento aplicado. La primera, permite entender la situación del colectivo trans en relación al ámbito laboral en el contexto capitalista neoliberal en la línea del trabajo de Irving (2008). La segunda, posibilita avanzar en construir un conocimiento transferible a las administraciones públicas con el fin de guiar sus actuaciones con respecto a la inserción laboral de las personas trans. Aunque a menudo ambas lógicas parezcan contradictorias, desde nuestro punto de vista es crucial poder combinar los análisis críticos de conjunto con aportaciones prácticas que permitan transformar el contexto en el que vivimos.

\section{REFERENCIAS BIBLIOGRÁFICAS}

Andréu, J. (2002). Las técnicas de análisis de contenido: una revisión actualizada. Documentos de Trabajo. Sevilla: Fundación Centro de Estudios Andaluces.

Barclay, J. M., y Scott, L. J. (2006). Transsexuals and workplace diversity-A case of "change" management. Personnel Review, 35(4): 487-502.

Budge, S. L., Tebbe, E. N., y Howard, K. A. (2010). The work experiences of transgender individuals: Negotiating the transition and career decision-making processes. Journal of Counseling Psychology, 57(4), 377-94.

Coll-Planas, G. (2010), La voluntad y el deseo. La construcción social del género y la sexualidad. El caso de lesbianas, gays y trans. Madrid/Barcelona: Egales.

Coll-Planas, G. y Cruells, M. (2013). "La puesta en práctica de la interseccionalidad política: el caso de las políticas LGTB en Cataluña". Revista Española de Ciencia Política, 31: 153-172.

De Cuypere, G. y Olyslager, F. (2009). 'Genderidentiteitsstoornissen: nieuwe visies en trends in de behandeling aan het UZGent', en: Verslagen van het Centrum voor Genderstudies-UGent 18, Ghent: Academia Press: 23-37.

Dietert, M., y Dentice, D. (2009). Gender identity issues and workplace discrimination: The transgender experience. Journal of Workplace Rights, 14(1): 121-140.

Espluga, J., Baltiérrez, J., y Lemkow, L. (2004). Relaciones entre la salud, el desempleo de larga duración y la exclusión social de los jóvenes en España. Cuadernos de trabajo social, 17, 45-62.

Fernández Dávila, P., y Morales, A. (2011). Estudio TranSex 2010. Conductas de riesgo y detección de necesidades para la prevención del VIH/ITS en mujeres transexuales trabajadoras sexuales. Barcelona: Stop Sida. 
FRA [Fundamental Rights Agency] (2014). Being Trans in the European Union. Comparative analysis of EU LGBT survey data. Luxemburg: Publications Office of the European Union.

Gates, G. J. (2011). How many people are lesbian, gay, bisexual and transgender? Los Angeles: The Williams Institute.

Halberstam, J. (2005). Unlosing Brandon: Brandon Teena, Billy Tipton, and Transgender Biography. In a Queer Time and Place: Transgender Bodies, Subcultural Lives. New York: NYU Press, 47-75.

Irving, D. (2008). Normalized transgressions: Legitimizing the transsexual body as productive. Radical History Review, 100, 38-59.

Izquierdo, María Jesús (1998). El malestar en la desigualdad. Madrid: Cátedra.

Juliano, D. (2004). Excluidas y marginales: una aproximación antropológica. Madrid: Cátedra.

Kuiper, A.J. (1991). Transseksualiteit: evaluatie van de geslachtsaanpassende behandeling. Utrecht: Elinkwijk.

Law, C. L., Martinez, L. R., Ruggs, E. N., Hebl, M. R., y Akers, E. (2011). Trans-parency in the workplace: How the experiences of transsexual employees can be improved. Journal of Vocational Behavior, 79(3), 710-723.

Lehtonen, J. y Mustola K. (2004). Straight people don't tell, do they? Negotiating the boundaries of sexuality and gender at work. Helsinki: Ministry of Labor.

Lombardi, E. L., Wilchins, R. A., Priesing, D., y Malouf, D. (2001). Gender violence: Transgender experiences with violence and discrimination. Journal of Homosexuality, 42, 89-101.

Mejía, N. (2006). Transgenerismos: una experiencia transexual desde la perspectiva antropológica. Barcelona: Bellaterra.

McNeil, J., Bailey, L., Ellis, S., Morton, J., y Regan, M. (2012). Trans mental health study 2012. Edinburgh: Scottish Transgender Alliance.

Missé, Miquel (2013). Transexualidades: otras miradas posibles. Barcelona: Editorial Egales,

Monro, S., y Warren, L. (2004). Transgendering citizenship. Sexualities, 7(3), 345-362.

Motmans, J., de Biolley, I., y Debunne, S. (2010). Being transgender in Belgium: Mapping the social and legal situation of transgender people. Bruxelles: Institute for the equality of woman and men.

Mulder, H.W. (1992). Gevraagd: werknemer m/v: een onderzoek naar de arbeidsparticipatie van transsexuelen voor en na de geslachtsaanpassende behandeling. Utrecht: SGG.

Platero, R. (Lucas) (2009). Transexualidad y agenda política: una historia de (dis) continuidades y patologizacion. Política y sociedad, 46(1/2): 107-128.

Platero, R. (Lucas) (ed.) (2013), Intersecciones: cuerpos y sexualidades en la encrucijada. Barcelona: Bellaterra.

Ruiz Olabuénaga, J. I. (1996), Metodología de la investigación cualitativa. Bilbao: Deusto. Schilt, K. (2006). Just one of the guys? How transmen make gender visible at work. Gender and Society, 20(4): 465-490. 
Schilt, K., y Connell, K. (2007). Do workplace gender transitions make gender trouble? Gender, Work and Organization, 14(6): 596-618.

Schilt, K., y Wiswall, M. (2008). Before and after: Gender transitions, human capital, and workplace experiences. The BE Journal of Economic Analysis E Policy, 8(1).

Serrano Argüello, N. (2011). La edad como factor de discriminación en el empleo. Revista Universitaria de Ciencias del Trabajo, 12, 17-43.

Soley-Beltran, P. y Coll-Planas, G. (2011), "'Having Words for Everything'. Institutionalising Gender Migration in Spain (1998-2008)", Sexualities, 3(14): 334-353.

STA (2008). Transgender experiences in Scotland. Key research findings of the Scottish Transgender Alliance survey of transgender people living in Scotland. Edinburgh: Scottish Transgender Alliance.

Torns, T., y Recio, C. (2012). Las desigualdades de género en el mercado de trabajo: entre la continuidad y la transformación. Revista de economía crítica, 14, 178-202.

van Kesteren, P. J., Gooren, L. J., y Megens, J. A. (1996). An epidemiological and demographic study of transsexuals in the Netherlands. Archives of sexual behavior, 25(6), 589-600.

Vartabedian, J. (2014). Migraciones trans: travestis brasileñas migrantes trabajadoras del sexo en Europa. cadernos pagu, 42: 275-312.

WPATH (2012). Normas de atención para la salud de personas trans y con variabilidad de género. https://amo_hub_content.s3.amazonaws.com/Association140/files/SOC\% 20-\%20Spanish\%20v2.pdf (consultado julio 2017).

Whittle, S., Turner, L., Al-Alami, M., Rundall, E., y Thom, B. (2007). Engendered penalties: Transgender and transsexual people's experiences of inequality and discrimination. Wetherby: Communities and Local Government publications.

Whittle, S., Turner, L., Coombs, R., y Rhodes, S. (2008). Transgender Eurostudy: Legal survey and focus on the transgender experience of health care. Bruxelles: ILGA Europe.

GERARD COLL PLANAS es Doctor en Sociología por la Universidad Autónoma de Barcelona (Mención de Doctor europeo) y es profesor titular en la Universidad de Vic - Universidad Central de Catalunya, miembro del grupo de investigación consolidado (AGAUR SGR - 833) Estudios de género: traducción, literatura, historia y comunicación". Es coordinador académico del proyecto 'Connected Equalities. Interseccionality in local public policies', cofinanciado por la Comisión Europea, y co-IP de 'Miradas poliédricas a la violencia de género. Propuestas para la prevención en los centros de secundaria desde una perspectiva interseccional' (convocatoria Recercaixa). Ha publicado artículos académicos en Sexualities, Athenea Digital, European Journal of Women's Studies, Nouvelles Questions Féministes, Revista Española de Ciencia Política y Papers. Revista de Sociologia.

http://orcid.org/0000-0002-2294-2707 
MIQUEL MISSÉ es sociólogo por la Universidad Autónoma de Barcelona (UAB) e investigador en la Universidad de Vic - Universidad Central de Catalunya. Ha participado en diversos proyectos de investigación sobre el colectivo trans y se dedica a la formación y asesoramiento en temas trans, género y sexualidad. Es coordinador y asesor desde hace 10 años el Espacio Abierto Trans-Intersex de Barcelona, espacio de encuentro de personas trans y su entorno. Así como promotor de diversas iniciativas de sensibilización en torno a la cuestión trans. Ha sido formador en el Máster de Sexualidad Humana, en el Máster de Sexualidad Clínica del Instituto de Estudios de la Sexualidad y la Pareja, en Barcelona Activa, en el Institut Català de la Salut, a la Consejería de Educación de la Generalidad Valenciana, en el Observatorio para la Igualdad de la UAB y en la Universidad de Verano de la UAB.

Recibido: 26-07-2017

Aceptado: 12-03-2018

\section{(c) (i) Licencia Creative Commons Reconocimiento (CC BY 4.0)}

